\title{
Eulerian Statistically Preserved Structures in Passive Scalar Advection
}

\author{
Yoram Cohen ${ }^{1,2}$ Anna Pomyalov ${ }^{1}$ and Itamar Procaccia ${ }^{1,2}$ \\ 1 Dept. of Chemical Physics, The Weizmann Institute of Science, Rehovot 76100, Israel. \\ 2 Dept. of Physics, the Chinese University of Hong Kong, Shatin, Hong Kong.
}

\begin{abstract}
We analyze numerically the time-dependent linear operators that govern the dynamics of Eulerian correlation functions of a decaying passive scalar advected by a stationary, forced 2-dimensional Navier-Stokes turbulence. We show how to naturally discuss the dynamics in terms of effective compact operators that display Eulerian Statistically Preserved Structures which determine the anomalous scaling of the correlation functions. In passing we point out a bonus of the present approach, in providing analytic predictions for the time-dependent correlation functions in decaying turbulent transport.
\end{abstract}

\section{INTRODUCTION}

The aim of this paper is to discuss the statistical physics of turbulent advection of passive scalars [1]. We are interested in scalar fields $\theta(\boldsymbol{r}, t)$ which are advected by a velocity field $\boldsymbol{u}(\boldsymbol{r}, t)$ such that together they solve the set of equations

$$
\begin{aligned}
& \frac{\partial \boldsymbol{u}}{\partial t}+(\boldsymbol{u} \cdot \nabla) \boldsymbol{u}=-\nabla p+\nu \Delta \boldsymbol{u}+\boldsymbol{f} \\
& \frac{\partial \theta}{\partial t}+(\boldsymbol{u} \cdot \nabla) \theta=\kappa \Delta \theta+f
\end{aligned}
$$

In these equations $p(\boldsymbol{r}, t), \nu$ and $\kappa$ are the pressure field, the kinematic viscosity and the scalar diffusivity respectively. In this paper the Navier-Stokes equations will be always forced with a time-stationary forcing $\boldsymbol{f}(\boldsymbol{r}, t)$. The scalar field will be forced or unforced (decaying), depending on our interests below. We focus on the case of high Reynolds number Re and high Peclet number Pe, i.e. $\nu, \kappa \rightarrow 0$, where the turbulence of the velocity field is fully developed and where both fields display a significant range of scaling behavior at scales that are sufficiently far from the forcing scales.

A major theoretical question that had been answered recently has to do with the scaling properties of the correlation functions of the advected field [2, 3]. Define the simultaneous many-point correlation function $F^{(N)}\left(\boldsymbol{r}_{1}, \ldots \boldsymbol{r}_{N}\right)$ in the forced case by

$$
F^{(N)}\left(\boldsymbol{r}_{1}, \ldots \boldsymbol{r}_{N}\right) \equiv\left\langle\theta\left(\boldsymbol{r}_{1}, t\right) \theta\left(\boldsymbol{r}_{2}, t\right) \cdots \theta\left(\boldsymbol{r}_{N}, t\right)\right\rangle_{f},
$$

with $\langle\cdots\rangle_{f}$ denoting an average with respect to realizations of the stationary forcing $f(\boldsymbol{r}, t)$ and of the velocity field $\boldsymbol{u}(\boldsymbol{r}, t)$. It had been known for a long time that for high Re and Pe these functions are homogeneous functions of their arguments, i.e.

$$
F^{(N)}\left(\lambda \boldsymbol{r}_{1}, \ldots \lambda \boldsymbol{r}_{N}\right)=\lambda^{\zeta_{N}} F^{(N)}\left(\boldsymbol{r}_{1}, \ldots \boldsymbol{r}_{N}\right)
$$

with $\zeta_{N}$ being a scaling exponent that in general is anomalous, i.e. cannot be guessed from dimensional considerations. But only recently it became clear how these exponents are determined by the dynamical processes.
To understand the progress made, we rewrite the dynamical equation for $\theta(\boldsymbol{r}, t)$ in the shorthand form

$$
\frac{\partial \theta(\boldsymbol{r}, t)}{\partial t}=\mathcal{L} \theta(\boldsymbol{r}, t)+f(\boldsymbol{r}, t),
$$

where in the present case $\mathcal{L} \equiv \boldsymbol{u} \cdot \boldsymbol{\nabla}-\kappa \Delta$. In recent work [4, 5] it was clarified why and how passive fields exhibit anomalous scaling, when the velocity field is a generic turbulent field. The key is to consider a decaying problem associated with Eq. (5), in which the forcing $f(\boldsymbol{r}, t)$ is put to zero. The problem becomes then a linear initial value problem,

$$
\partial \theta / \partial t=\mathcal{L} \theta
$$

with a formal solution

$$
\theta(\boldsymbol{r}, t)=\int d \boldsymbol{r}^{\prime} R\left(\boldsymbol{r}, \boldsymbol{r}^{\prime}, t\right) \theta\left(\boldsymbol{r}^{\prime}, 0\right),
$$

with the operator

$$
\left.R\left(\boldsymbol{r}, \boldsymbol{r}^{\prime}, t\right) \equiv T^{+} \exp \left[\int_{0}^{t} d s \mathcal{L}(s)\right]\right|_{\boldsymbol{r}, \boldsymbol{r}^{\prime}},
$$

and $T^{+}$being the time ordering operator. Define next the time dependent correlation functions of the decaying problem:

$$
C^{(N)}\left(\boldsymbol{r}_{1}, \cdots, \boldsymbol{r}_{N}, t\right) \equiv\left\langle\theta\left(\boldsymbol{r}_{1}, t\right) \cdots \theta\left(\boldsymbol{r}_{N}, t\right)\right\rangle .
$$

Here pointed brackets without subscript $f$ refer to the decaying object in which averaging is taken with respect to realizations of the velocity field and initial conditions. As a result of Eq. (7) the decaying correlation functions are evolved by a propagator $\mathcal{P}^{(N)}(\underline{\boldsymbol{r}}, \underline{\boldsymbol{\rho}}, t)$, (with $\underline{\boldsymbol{r}} \equiv \boldsymbol{r}_{1}, \boldsymbol{r}_{2}, \cdots, \boldsymbol{r}_{N}$ and $\left.\underline{\boldsymbol{\rho}} \equiv \boldsymbol{\rho}_{1}, \boldsymbol{\rho}_{2}, \cdots, \boldsymbol{\rho}_{N}\right)$ :

$$
C^{(N)}(\underline{\boldsymbol{r}}, t)=\int d \underline{\boldsymbol{\rho}} \mathcal{P}^{(N)}(\underline{\boldsymbol{r}}, \underline{\boldsymbol{\rho}}, t) C^{(m)}(\underline{\boldsymbol{\rho}}, 0) .
$$

In writing this equation we made explicit use of the fact that the initial distribution of the passive field $\theta(\boldsymbol{r}, 0)$ is statistically independent of the advecting velocity field. Thus the operator $\mathcal{P}^{(N)}(\underline{\boldsymbol{r}}, \underline{\boldsymbol{\rho}}, t)$ can be written explicitly

$\mathcal{P}^{(N)}(\underline{\boldsymbol{r}}, \underline{\boldsymbol{\rho}}, t) \equiv\left\langle R\left(\boldsymbol{r}_{1}, \boldsymbol{\rho}_{1}, t\right) R\left(\boldsymbol{r}_{2}, \boldsymbol{\rho}_{2}, t\right) \cdots R\left(\boldsymbol{r}_{N}, \boldsymbol{\rho}_{N}, t\right)\right\rangle$. 
The key finding [4, 5] is that the operator $\mathcal{P}^{(N)}(\underline{\boldsymbol{r}}, \underline{\boldsymbol{\rho}}, t)$ possesses a left eigenfunction of eigenvalue 1 , i. e. there exists (for each $N$ ) a time-independent function $Z^{(N)}(\underline{\boldsymbol{r}})$ satisfying

$$
Z^{(N)}(\underline{\boldsymbol{r}})=\int d \underline{\boldsymbol{\rho}} Z^{(N)}(\underline{\boldsymbol{\rho}}) \mathcal{P}^{(N)}(\underline{\boldsymbol{r}}, \underline{\boldsymbol{\rho}}, t) .
$$

The functions $Z^{(N)}$ are referred to as "Statistically Preserved Structures", being invariant to the dynamics, even though the operator is strongly time dependent and decaying. How to form, from these functions, infinitely many conserved variables in the decaying problem was shown in [4], and is discussed again in Sect. III. The functions $Z^{(N)}(\underline{\boldsymbol{r}})$ are homogeneous functions of their arguments, with anomalous scaling exponents $\zeta_{N}$ :

$$
Z^{(N)}(\lambda \underline{\boldsymbol{r}})=\lambda^{\zeta_{N}} Z^{(N)}(\underline{\boldsymbol{r}})+\ldots
$$

where "..." stand for sub-leading scaling terms. Since Eq. (12) contains $Z^{(N)}(\underline{\boldsymbol{r}})$ on both sides, the scaling exponent $\zeta_{N}$ cannot be determined from dimensional considerations, and it can be anomalous. More importantly, it was shown that the correlation functions of the forced case, $F^{(N)}(\underline{\boldsymbol{r}})$ Eq. (3), have exactly the same scaling exponents as $Z^{(N)}(\underline{\boldsymbol{r}})[\underline{5}$. In the scaling sense

$$
F^{(N)}(\underline{\boldsymbol{r}}) \sim Z^{(N)}(\underline{\boldsymbol{r}})
$$

This is how anomalous scaling in passive fields is understood.

Besides exactly soluble examples in which the advecting velocity field is non-generic (i.e. $\delta$-correlated in time) the existence of eigenfunctions of eigenvalue 1 of the time dependent propagator was demonstrated fully only in shell models of turbulence. While the present authors believe that shell models contain a lot of the robust properties of real turbulence, this belief is not universally accepted in the community. It is therefore necessary to demonstrate that the mechanism sketched above exists indeed in the full problem Eqs. (11)-(2). This had been done for 3'rd order correlations within the Lagrangian formulation in [3]. The aim of this paper is to demonstrate this in the Eulerian frame, and for correlation functions of order 2, 4 and 6 .

In Section II we describe the simulations of Eqs. (1) and (2) and the type of measurements that we performed. Sect. III is a theoretical digression, in which we analyze an exactly soluble simple model to guide ourselves as to how to analyze the numerical results to find the scaling forms of the $n$th oder propagators and their eigenfunctions. Sect. IV describes the analysis of the data, and Sect. V offers a discussion and a summary of the paper.

\section{SIMULATIONS}

We performed a Direct Numerical Simulation (DNS) of Eqs. (11) and (2) on a $2048 \times 20482$-D array. The forcing $\boldsymbol{f}$ in Eq. (11) is random, $\delta$ correlated in time, isotropic and homogeneous. Its $k$ depdendence is

$$
\langle|\boldsymbol{f}(\boldsymbol{k})|\rangle \propto k \exp \left[-0.5(k / 1024)^{2}\right] .
$$

This forcing is biased towards the small scales; this is done because of the inverse energy cascade that characterizes 2-dimensional turbulence. The fluid dissipation is modeled by a hyper-viscosity term proportional to $\Delta^{8} \boldsymbol{u}$. In addition we employed a "friction" term proportional to $\boldsymbol{u}$ in order to stabilize the velocity field on the largest scales. The passive field $\theta$ dissipates normally as shown in Eq. (2).

The simulations were performed for a decaying passive field $\theta$, that is, the forcing $f$ in Eq. (2) was put to zero. The initial conditions for the $\theta$ field were of the form

$$
\tilde{\theta}(\boldsymbol{k}, t=0)=\delta\left(k-k_{0}\right) \exp (i \gamma(\boldsymbol{k})),
$$

where $\tilde{\theta}(\boldsymbol{k}, t)$ is the Fourier transform of the real space variable defined as:

$$
\tilde{h}(\boldsymbol{k})=\frac{1}{2 \pi} \int d \boldsymbol{r} h(\boldsymbol{r}) e^{-i \boldsymbol{k} \boldsymbol{r}}
$$

(from now on we will omit the tilde above the functions and denote the $\mathrm{k}$ space functions only by their variables). $\gamma(\boldsymbol{k})$ is a random variable in the interval $\gamma \in[0,2 \pi]$ where $\gamma(\boldsymbol{k})=-\gamma(-\boldsymbol{k})$, insuring that $\theta^{*}(-\boldsymbol{k}, 0)=\theta(\boldsymbol{k}, 0)$, and therefore that $\theta(\boldsymbol{r}, t)$ is real.

As the initial conditions for $\theta$ and the forcing of the $\boldsymbol{u}$ fields are both homogeneous and isotropic so are the scalar correlation functions $C^{(N)}\left(\boldsymbol{r}_{1} \ldots \boldsymbol{r}_{N}, t\right)$ defined in Eq. (9), at all times $t$. We measured the $\boldsymbol{k}$ space correlation functions:

$$
\begin{aligned}
& C^{(N)}\left(\boldsymbol{k}_{1}, \ldots, \boldsymbol{k}_{N}, t\right) \delta\left(\boldsymbol{k}_{1}+\ldots+\boldsymbol{k}_{N}\right) \\
& =\left\langle\theta\left(\boldsymbol{k}_{1}, t\right) \ldots \theta\left(\boldsymbol{k}_{N}, t\right)\right\rangle
\end{aligned}
$$

where again the average $\langle\ldots\rangle$ is over initial conditions and over realizations of the $\boldsymbol{u}$ field. The delta function appears due to translational invariance.

In accordance to Eq. (10) we define the $\boldsymbol{k}$ space propagator:

$$
C^{(N)}(\underline{\boldsymbol{k}}, t)=\int d \underline{\boldsymbol{k}}^{\prime} \mathcal{P}^{(N)}\left(\underline{\boldsymbol{k}}, \underline{\boldsymbol{k}}^{\prime}, t\right) C^{(N)}\left(\underline{\boldsymbol{k}}^{\prime}, 0\right) .
$$

\section{A. The 2-point propagator}

The only two point correlator that is not zero is $C^{(2)}(\boldsymbol{k},-\boldsymbol{k}, t)=\left\langle\theta(\boldsymbol{k}) \theta^{*}(\boldsymbol{k})\right\rangle$. Because of the isotropy of the initial conditions and the driving field, the correlator depends only on the magnitude of $\boldsymbol{k}$. We can therefore consider the second order structure function $S^{(2)}(k, t)=$ $C^{(2)}(\boldsymbol{k},-\boldsymbol{k}, t)$, and it's propagator $\hat{\mathcal{P}}^{(2)}\left(k, k^{\prime}, t\right)$.

In discrete $k$-space (as in our simulation on a grid) the propagator $\hat{\mathcal{P}}^{(2)}\left(k, k^{\prime}, t\right)$ has a matrix representation. For 


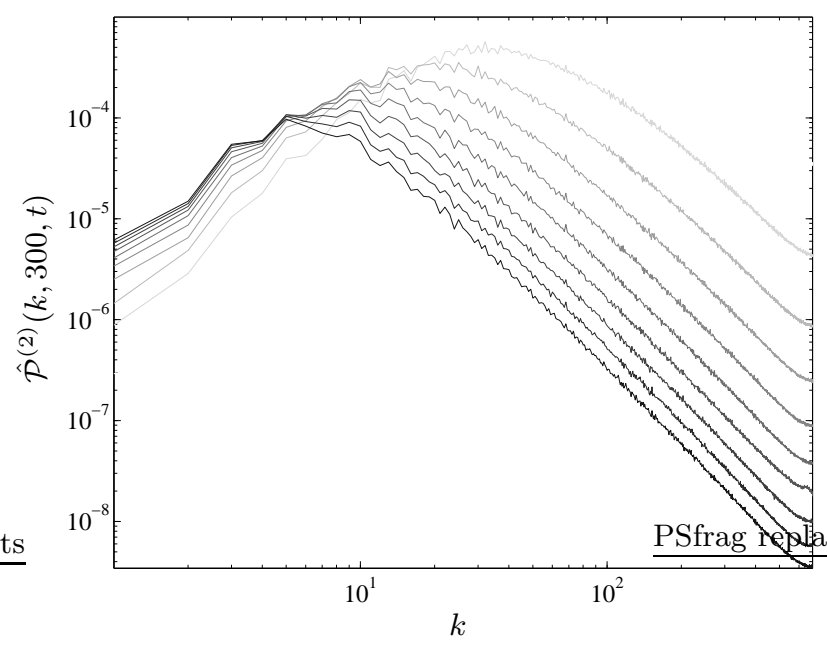

FIG. 1: $\hat{\mathcal{P}}^{(2)}(k, 300, t)$ for 10 different times. The first time is $\tau_{30}$, i.e. the eddy turn over time at scale $k=30$, in lightest grey. Later times, in darker and darker greys, are at $2 \tau_{30}$, $3 \tau_{30}$, etc. until $10 \tau_{30}$.

the choice of initial conditions as in Eq. (16), $S^{(2)}(k, t)$ is simply the $k_{0}$ th column of the propagator :

$S^{(2)}(k, t)=\int d k^{\prime} \hat{\mathcal{P}}^{(2)}\left(k, k^{\prime}, t\right) S^{(2)}\left(k^{\prime}, 0\right)=\hat{\mathcal{P}}^{(2)}\left(k, k_{0}, t\right)$.

In Figure 1 we plot such a column of the 2-point propagator, at 10 different times. Two properties of the propagator should be noticed: as time progresses the overall amplitude decreases due to the decay, while the maximum moves from the initial $k_{0}$ to lower values of $k$. In Sects. III and IV we will find the scaling form of this propagator.

\section{B. Multi-point propagators}

In the case of the multi-point correlator the overall $\delta$ function and the isotropy of the fields do not reduce the dependence to a one variable function. The propagators are therefore functions of many $k$-vectors. It turns out however that measurement of the statistics for objects depending on many $k$-vectors are very taxing. We opted therefore the extract from the DNS partial information on the dynamics of the $2 N$ order structure functions with $N>1$ :

$$
S^{(2 N)}(k, t)=\left\langle|\theta(\boldsymbol{k}, t)|^{2 N}\right\rangle .
$$

Accordingly we define the reduced propagators :

$$
S^{(2 N)}(k, t)=\int d k^{\prime} \hat{\mathcal{P}}^{(2 N)}\left(k, k^{\prime}, t\right) S^{(2 N)}\left(k^{\prime}, 0\right) .
$$

In Figure 2 we present the numerical results for of the fourth and sixth order reduced propagators, at the same ten different times. These objects display similar qualitative behavior to that of the 2-point propagator. We
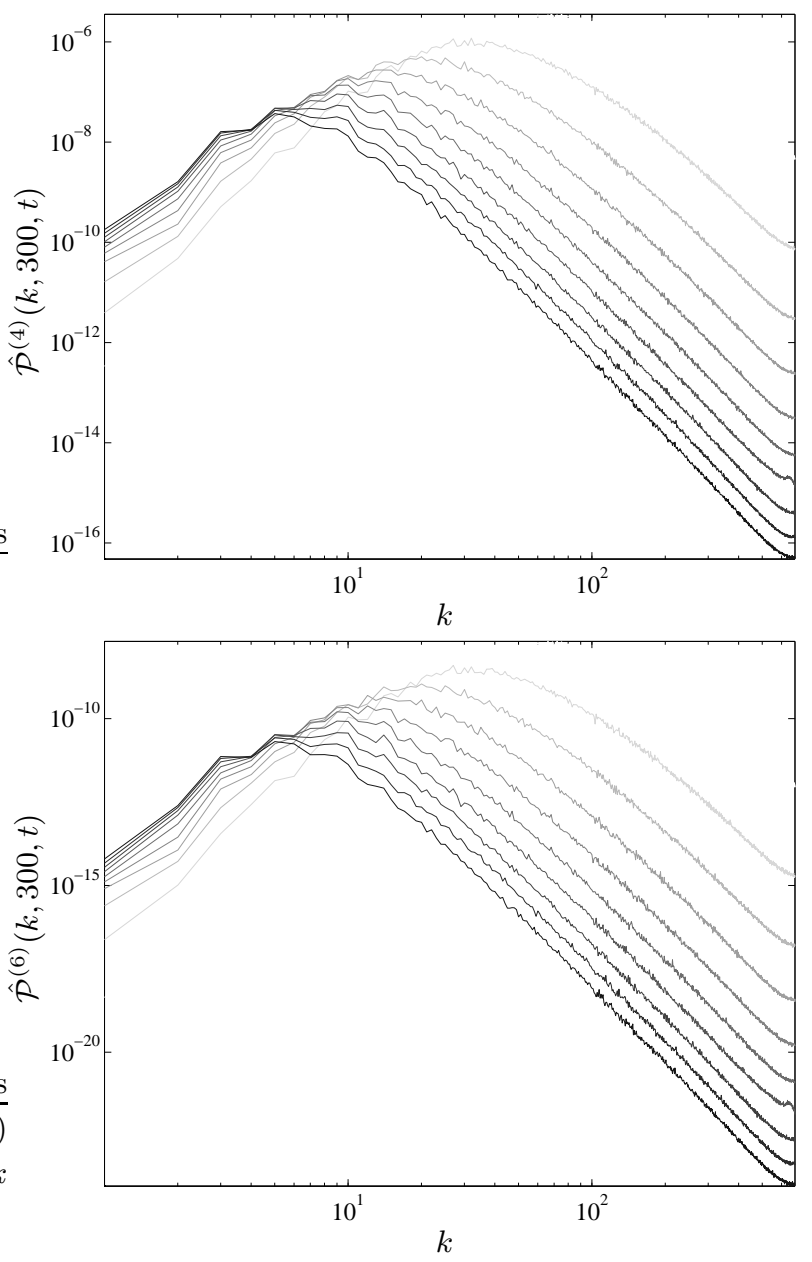

FIG. 2: $\hat{\mathcal{P}}^{(4)}(k, 300, t)$ (upper panel) and $\hat{\mathcal{P}}^{(6)}(k, 300, t)$ (lower panel) for the same 10 different times as in Fig. 1]

will learn in the next section how to think about the scaling properties of these objects and how to re-plot the numerical results in proper rescaled variables.

\section{AN EXACTLY SOLVABLE CASE}

In order to motivate the data analysis presented in Sect. IV we turn now to the Kraichnan model for passive scalar advection. The model is exactly solvable, and examining the analytic forms of the propagators offers clues to what should be expected in the generic case. In principle we could perform this analysis in terms of the Kraichnan model for a 2-dimensional passive scalar. In fact, it is sufficient to consider the shell-model version; the latter is very transparent to analytic manipulations, and for our purposes the results throw equally useful light on how to perform the analysis of the DNS results. 


\section{A. The Kraichnan shell model}

The Kraichnan shell model for passive scalar advection [4, 6], as all other shell models of turbulent flows, is written in terms of Fourier components of the field. The Fourier components are restricted to shells denoted by the index $m$, and the equation takes on the form

$$
\begin{aligned}
\frac{d \theta_{m}}{d t} & =\mathcal{L}_{m, n} \theta_{n} \\
\mathcal{L}_{m, n} & =i k_{m+1} u_{m+1} \delta_{m+1, n}+i k_{m} u_{m}^{*} \delta_{m-1, n}-\kappa k_{m}^{2} \delta_{m, n} .
\end{aligned}
$$

Here $k_{m}$ are the shell $k$ vector, $k_{m}=k_{0} \lambda^{m}$ for some $k_{0}$ and $\lambda$. The shell components of the velocity field, $u_{m}(t)$, are Gaussian random variables, $\delta$-correlated in time, satisfying:

$$
\left\langle u_{n}(t) u_{m}^{*}\left(t^{\prime}\right)\right\rangle=c_{0} \delta_{n, m} \delta\left(t-t^{\prime}\right) \lambda^{-\xi m} .
$$

Here $\xi$ is the scaling exponent of the $u$ field.

\section{B. The 2nd order propagator}

The second order correlator satisfies the explicit equation (see [5]):

$$
\frac{d}{d t} S_{n}^{(2)}(t)=\left(M_{n, m}^{(2)}-\kappa k_{n}^{2}\right) S_{m}^{(2)}(t),
$$

$S_{n}^{(2)}(t)$ is the shell equivalent of the 2 nd order structure function in Eq. (20),

$$
S_{n}^{(2)}(t)=\left\langle\left|\theta_{n}(t)\right|^{2}\right\rangle
$$

The time evolution operator $\boldsymbol{M}^{(2)}$ has the form

$$
\begin{aligned}
M_{n, m}^{(2)} & =\left(\alpha_{n}+\alpha_{n+1}\right) \delta_{n, m} \\
& -\alpha_{n} \delta_{n-1, m}-\alpha_{n+1} \delta_{n+1, m},
\end{aligned}
$$

where

$$
\alpha_{n} \equiv-c_{0} k_{0}^{2}\left(\frac{k_{n}}{k_{0}}\right)^{\zeta_{2}}=-c_{0} k_{0}^{2} \lambda^{\zeta_{2} n}
$$

and $\zeta_{2}=2-\xi$ is the dimensional scaling of the 2-point correlation function. The operator $\boldsymbol{M}^{(2)}$ has the following useful scaling property:

$$
\lambda^{-\zeta_{2} p} M_{n+p, m+p}^{(2)}=M_{n, m}^{(2)} .
$$

The second order propagator has, in the limit of vanishing viscosity, the explicit form:

$$
\mathcal{P}_{n \mid m}^{(2)}(t)=\left.\exp \left(t \boldsymbol{M}^{(2)}\right)\right|_{n, m}
$$

Note that the time evolution operator and the propagator are both Hermitian, and thus admit an eigenvector decomposition. The time evolution operator has two types of eigen-vectors $\psi_{n}^{(2, q)}$ and $\tilde{\psi}_{n}^{(2, q)}$ which we can regard as slow modes and fast modes respectively. Here the index $k$ stands for the eigen-mode index. The fast modes are dominated by the viscous term, their support is in the viscous range, and they can essentially be taken to be $\tilde{\psi}_{n}^{(2, q)}=\delta_{n, q}$ for some shell $q$ with the shell in$\operatorname{dex} q>m_{d}$, above which viscosity dominates Eq. (25). The transition shell $m_{d}$ is determined by the condition $\kappa k_{m_{d}}^{2}=c_{0} k_{m_{d}}^{\zeta_{2}}$. For the fast modes we have the following approximate Equation:

$$
\frac{d}{d t} \tilde{\psi}_{n}^{(2, q)}=-\kappa k_{n}^{2} \tilde{\psi}_{n}^{(2, q)},
$$

therefore their eigen-values are $\beta_{q}=-\kappa k_{q}^{2}$.

For slow modes, $\psi_{n}^{(2, q)}$, which have their support on shells smaller than $m_{d}$, the dissipative term can be neglected and they satisfy an eigen-function equation of the form:

$$
\beta_{q} \psi_{n}^{(2, q)}=M_{n, m}^{(2)} \psi_{m}^{(2, q)}
$$

For a sufficiently large inertial range we can use the scaling property (29) to get:

$$
\beta_{q} \psi_{n}^{(2, q)}=\lambda^{-\zeta_{2} p} M_{n+p, m+p}^{(2)} \psi_{m}^{(2, q)} .
$$

Shifting indices we rewrite,

$$
\beta_{q} \psi_{n-p}^{(2, q)}=\lambda^{-\zeta_{2} p} M_{n, m}^{(2)} \psi_{m-p}^{(2, q)}
$$

Define now a vector

$$
\chi_{n} \equiv \psi_{n-p}^{(2, q)}
$$

we have:

$$
\lambda^{\zeta_{2} p} \beta_{q} \chi_{n}=M_{n, m}^{(2)} \chi_{m}
$$

We can thus define $\psi_{n}^{(2, q+p)} \equiv \chi_{n}$, an eigen-function of the time evolution operator $\boldsymbol{M}^{(2)}$ with eigen-value $\lambda^{\zeta_{2} p} \beta_{q}$.

We can thefore conclude that the eigen-vectors and eigen-values may be obtained from each other by shift of the indices:

$$
\begin{aligned}
\psi_{n+p}^{(2, q+p)} & =\psi_{n}^{(2, q)} \\
\beta_{q+p} & =\lambda^{\zeta_{2} p} \beta_{q} .
\end{aligned}
$$

Fig. 3] demonstrates that indeed two different eigenfunctions coincide once shifted with respect to each other. It follows from (38) that

$$
\beta_{q} \propto \alpha_{q}
$$

In Fig. 4 the spectrum of the eigen-values is plotted, showing the two expected regions: a region for which the scaling of the eigen-values is $\propto k_{n}^{\zeta_{2}}$ for the slow modes, and $\propto k_{n}^{2}$ for the fast modes. The lowest eigenvalue 


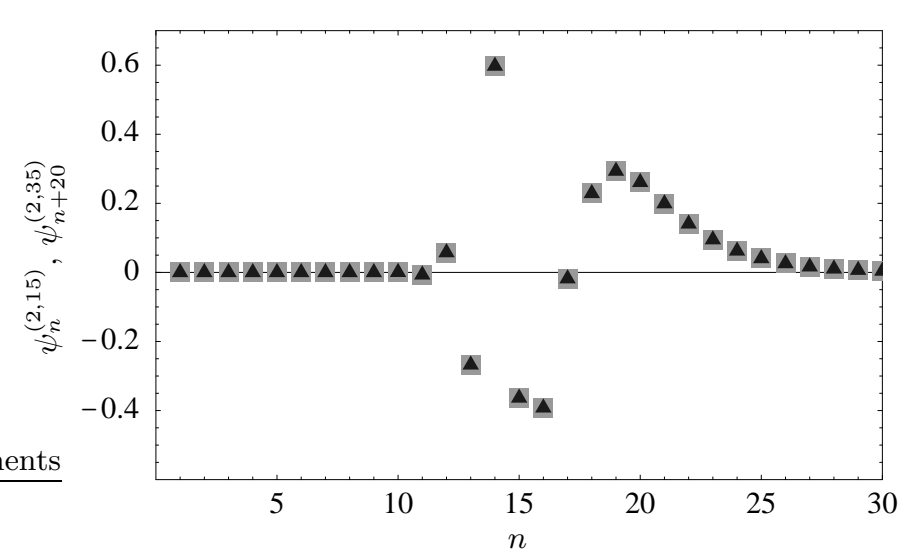

FIG. 3: A plot of the eigen-vectors $\psi_{n}^{2,15}$ (squares), and $\psi_{n+20}^{2,35}$ (triangles), for $n \in[0,30]$

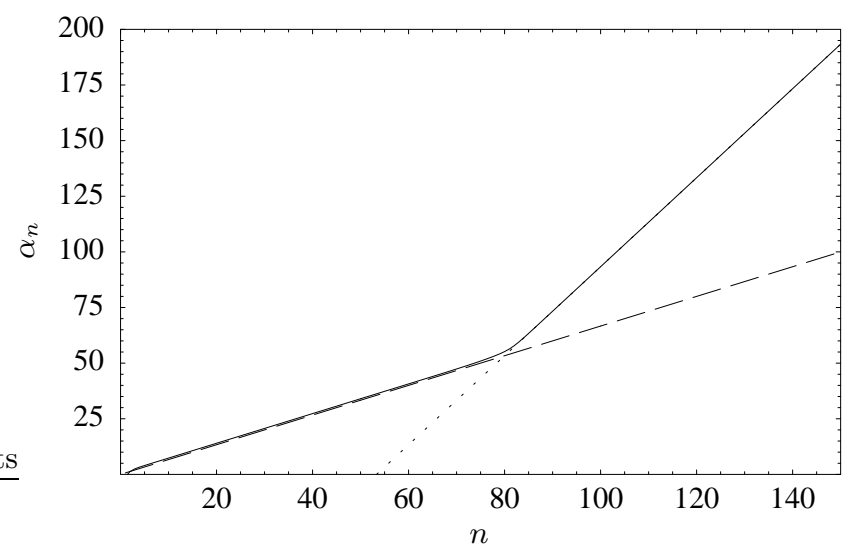

FIG. 4: The eigen-values, plotted from small to large. The eigen-values are well fitted by the analytic predictions $k_{m}^{\zeta_{2}}$ (dashed line) for the slow modes, and by $k_{m}^{2}$ (dotted line) for the fast modes. The transition to viscous range occur at shell $m_{d}=80$

is proportional to $\alpha_{0}$. The eigen-function $\psi_{n}^{(2,0)}$ associated with it can be calculated explicitly, and it exhibits a normal, dimensional, scaling in the inertial range:

$$
\psi_{n}^{(2,0)} \propto\left(k_{n} / k_{0}\right)^{-\zeta_{2}} .
$$

Using Eqs (37) and (40), that imply that $\psi_{n}^{(2, q)}$ has a scailing 'tail' with exponent $\zeta_{2}$, starting after shell $q$ we establish:

$$
\psi_{n}^{(2, q)} \propto\left(k_{n} / k_{q}\right)^{-\zeta_{2}} \propto \alpha_{q} / \alpha_{n} \quad q<n<m_{d},
$$

We now use these results to learn how to re-scale our numerical data. Suppose that we started with an initial condition in some shell $n$ in the inertial range, $S_{p}^{(2)}(0)=$ $\delta_{p, n}$. The fast modes do not contribute significantly to the time dependence, since they decay fast and anyhow have no support in the inertial range. Thus, using only the slow modes and Eqs. (27), (30), we have for $p<n$,

$$
\begin{aligned}
S_{p}^{(2)}(t) & =\sum_{q=1}^{p} \psi_{n}^{(2, q)} e^{\alpha_{q} t} \psi_{p}^{(2, q)} \\
& \propto \frac{1}{\alpha_{p} \alpha_{n}} \sum_{q=1}^{p} \alpha_{q}^{2} e^{\alpha_{q} t} \propto \mathcal{P}_{p \mid n}^{(2)}(t) .
\end{aligned}
$$

In going from the first to the second line we inserted the $\delta$-function initial conditions, getting thus a column of the propagator $\mathcal{P}_{p \mid n}^{(2)}(t)$. Remember that in our DNS calculations we fixed the column index, and are interested in the scaling behavior with respect to the row index and time. Therefore we compute now (up to an overall dimensional constant),

$$
\begin{aligned}
& \lambda^{\zeta_{2} m} \mathcal{P}_{p-m \mid n}^{(2)}\left(\lambda^{\zeta_{2} m} t\right)=\frac{\lambda^{\zeta_{2} m}}{\alpha_{p-m} \alpha_{n}} \sum_{q=1}^{p-m} \alpha_{q}^{2} e^{\alpha_{q+m} t} \\
& =\frac{1}{\alpha_{p} \alpha_{n}} \sum_{q=1}^{p-m} \alpha_{q+m}^{2} e^{\alpha_{q+m} t}=\frac{1}{\alpha_{p} \alpha_{n}} \sum_{q=m+1}^{p} \alpha_{q}^{2} e^{\alpha_{q} t} \\
& =\mathcal{P}_{p \mid n}^{(2)}(t)-\frac{\alpha_{m}}{\alpha_{p}} \mathcal{P}_{m \mid n}^{(2)}(t)
\end{aligned}
$$

where we have used the explicit form of the propagator in Eq. (42) and Eq. (28). For $m$ much smaller than $p$ we can neglect the second term. Then we conclude that the propagator is a homogeneous function of the variables $k_{p}$ and $t$. Explicitly, multiplying by $t$,

$$
t \mathcal{P}^{(2)}\left(k_{p}, k_{n}, t\right)=\left(\lambda^{-\zeta_{2} m} t\right) \mathcal{P}^{(2)}\left(\lambda^{m} k_{p}, k_{n}, \lambda^{-\zeta_{2} m} t\right)
$$

For fixed $k_{n}$ this is a homogeneous function of two arguments which can be always written in the form

$$
\mathcal{P}^{(2)}\left(k_{p}, k_{n}, t\right)=\frac{\text { Const }}{\alpha_{n} t} \Lambda\left(\alpha_{p} t\right),
$$

for some function $\Lambda(x)$. The symmetry between $p$ and $n$ is restored by realizing that the asymptotic form of $\Lambda(x)$ is $1 / x$ for large $x$.

This scaling form was also found in the study of the generic shell models of passive scalar advection (arbitrary time dependence in the velocity correlations) [5] and we will show that the same scaling form survives when we go back to the generic model studied by DNS.

\section{The multi point propagators}

The invariance of Eq. (24) under a uniform phase change $\theta_{n} \rightarrow e^{i \phi} \theta_{n}$ dictates that the only non-zero correlation functions will have an equal number of variables $\theta_{n}$ and conjugated variables $\theta_{m}^{*}$. We define:

$$
C_{i_{1}, \ldots, i_{m}, j_{1}, \ldots, j_{N}}^{(2 N)}(t)=\left\langle\theta_{i_{1}}(t) \ldots \theta_{i_{N}}(t) \theta_{j_{1}}^{*}(t) \ldots \theta_{j_{N}}^{*}(t)\right\rangle
$$


As in the 2-point case we can define the respective multipoint differential time derivative operator, analogous to Eq. (25). In the limit of vanishing viscosity:

$$
\frac{d}{d t} C_{\underline{\boldsymbol{i}}, \underline{\boldsymbol{j}}}^{(2 N)}(t)=M_{\underline{\boldsymbol{i}}, \underline{\boldsymbol{j}} \underline{i}^{\prime}, \underline{\boldsymbol{j}}^{\prime}}^{(2 N)} C_{\underline{\boldsymbol{i}}^{\prime}, \underline{\boldsymbol{j}}^{\prime}}^{(2 N)}(t)
$$

The respective propagator is defined by:

$$
\begin{aligned}
& C_{\underline{\boldsymbol{i}}, \underline{\boldsymbol{j}}}^{(2 N)}(t)=\mathcal{P}_{\underline{\boldsymbol{i}}, \underline{\boldsymbol{j}} \underline{\underline{\boldsymbol{i}}^{\prime}, \underline{\boldsymbol{j}}^{\prime}}}^{(2 N)}(t) C_{\underline{\boldsymbol{i}}^{\prime}, \underline{\boldsymbol{j}}^{\prime}}^{(2 N)}(0)
\end{aligned}
$$

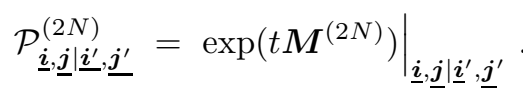

Because of the time reversibility of the statistics of the $\boldsymbol{u}$ fields, and the anti-hermiticity of the un-averaged differential operator $\mathcal{L}$ in Eq. (23): $\mathcal{L}_{m, n}^{*}=-\mathcal{L}_{n, m}$, both the time derivative operator $\boldsymbol{M}^{(2 N)}$ and the propagator itself are hermitian, and therefore admit eigenvalue decomposition. Furthermore the operator $\boldsymbol{M}^{(2 N)}$ has the following scaling property:

$$
M_{\underline{\boldsymbol{i}}, \underline{\boldsymbol{j}} \underline{\underline{\boldsymbol{i}}^{\prime}, \underline{\boldsymbol{j}}^{\prime}}}^{(2 N)}=\lambda^{-\zeta_{2} p} M_{\underline{\boldsymbol{i}}+p, \underline{\boldsymbol{j}}+p \mid \underline{\boldsymbol{i}}^{\prime}+p, \underline{\boldsymbol{j}}^{\prime}+p}^{(2 N)} .
$$

As in the two point case the dynamics within the scaling range is determined by slow modes.

$$
\beta_{k}^{(2 N, l)} \psi_{\underline{\boldsymbol{i}}, \underline{j}}^{(2 N, l, k)}=M_{\underline{\boldsymbol{i}}, \underline{j} \underline{\underline{i}}^{\prime}, \underline{j}^{\prime}}^{(2 N)} \psi_{\underline{\boldsymbol{i}}^{\prime}, \underline{\boldsymbol{j}}^{\prime}}^{(2 N, l, k)} .
$$

In this equation the index $l$ stands for a family of eigenmodes, and $k$ for their index within the family. Each $l$ family can be obtained from any one of its members by shifting the indices. The eigen-values of the modes within a given family can be obtained also by a shift :

$$
\begin{aligned}
\psi_{\underline{\boldsymbol{i}}+p, \underline{\boldsymbol{j}}+p}^{(2 N, l, k+p)} & =\psi_{\underline{\boldsymbol{i}}, \underline{\boldsymbol{j}}}^{(2 N, l, k)} \\
\beta_{k+p}^{(2 N, l)} & =\lambda^{\zeta_{2} p} \beta_{k}^{(2 N, l)} .
\end{aligned}
$$

Note that in the 2-point case we had only one family of eigen-modes. Here we added the index $l$ to the eigenvalues and eigen-modes to distinguish the different families. Note also that in Eq. (53) the scaling exponent of the eigenvalues is $\zeta_{2}$; this stems from the scaling properties of the differential operator $\boldsymbol{M}^{(2 N)}$, cf. eq. (50). However, the eigen-modes display in general anomalous scaling which can be represented by

$$
\psi_{\underline{\boldsymbol{i}}, \underline{\boldsymbol{j}}}^{(2 N, l, k)}=\lambda^{-p \zeta_{2 N, l}} \psi_{\underline{\boldsymbol{i}}+p, \underline{\boldsymbol{j}}+p}^{(2 N, l, k)},
$$

where $\zeta_{2 N, l}$ is the (anomalous) scaling exponent of the $l$ th family of eigen-modes.

Since the DNS were analyzed in terms of fused objects, we focus on initial conditions in Eq. (48) for which all the $2 N$ indices are the same. We also measure the resulting structure functions

$$
S_{n}^{(2 N)}=\left\langle\left|\theta_{n}\right|^{2 N}\right\rangle .
$$

This procedure will extract a fused propagator for which there are only two indices, and we denote it bellow as
$\mathcal{P}_{p \mid n}^{(2 N)}(t)$. This propagator is not Hermitian, it has in general no eigen-function decomposition, but we can understand the scaling form of any of its columns from the knowledge of the full propagator and its eigen-functions.

The equivalent of Eq. (42) for $S_{k}^{(2 N)}(t=0)=\delta_{k, n}$, $p<n$, summing over all families of slow modes (i.e. over the index $l$ ) is:

$$
\begin{aligned}
& S_{p}^{(2 N)}(t)=\sum_{l} \sum_{q=1}^{p} \psi_{n}^{(2 N, l, q)} e^{\alpha_{q} t} \psi_{p}^{(2 N, l, q)} \\
& =\sum_{l} \frac{C_{l}}{\left(\alpha_{p} \alpha_{n}\right)^{\frac{\zeta_{2 N, l}}{\zeta_{2}}}} \sum_{q=1}^{p} \alpha_{q}^{\frac{2 \zeta_{2 N, l}}{\zeta_{2}}} e^{\alpha_{q} t}=\mathcal{P}_{p \mid n}^{(2 N)}(t)
\end{aligned}
$$

In going from first to second line we have used the fact that

$$
\psi_{i, i, \ldots, i}^{2 N, l, p} \propto\left(\frac{\alpha_{i}}{\alpha_{p}}\right)^{-\zeta_{2 N, l} / \zeta_{2}} .
$$

This follows directly from Eqs. (52) and (54).

Using the same argumentation as in Eq. (43) we get:

$$
\mathcal{P}_{p \mid n}^{(2 N)}(t)=\sum_{l} \frac{C_{l}}{\left(\alpha_{n} t\right)^{\frac{\zeta_{2 N, l}}{\zeta_{2}}}} \Lambda^{(2 N, l)}\left(\alpha_{p} t\right),
$$

here again the $C_{l}$ 's are dimensional constants and the functions $\Lambda^{(2 N, l)}(x)$ are some functions with the asymptotic form $\Lambda^{(2 N, l)}(x) \approx x^{-\frac{\zeta_{2 N, l}}{\zeta_{2}}}$ for $x$ large. We note that for sufficiently long times and if the scaling exponents are well separated, we can expect the sum to be dominated by the leading scaling exponent.

\section{DATA ANALYSIS}

The detailed analysis that was possible for the simple model of Sect. III is not available for the generic model Eqs.(11), (2). Our strategy is to assume that the scaling forms derived in the last section are still valid for the generic model, and to demonstrate this by replotting the data accordingly. We will see that these predictions are born out by the data.

\section{A. analysis of the 2-point porpagator}

In light of Eq. (44), we expect the 2nd order propagator $\hat{\mathcal{P}}^{(2)}\left(k, k_{0}, t\right)$ to be, for a fixed $k_{0}$, a homogeneous function of the variable $k t^{1 / \zeta_{2}}$, and to decay as $1 / t$ :

$$
\hat{\mathcal{P}}^{(2)}\left(k, k_{0}, t\right) \propto \frac{1}{t} H^{(2)}\left(k t^{1 / \zeta_{2}}\right),
$$

where $H^{(2)}$ is some function. We test the correctness of this form in Fig. [5] To this aim we re-plot that data 


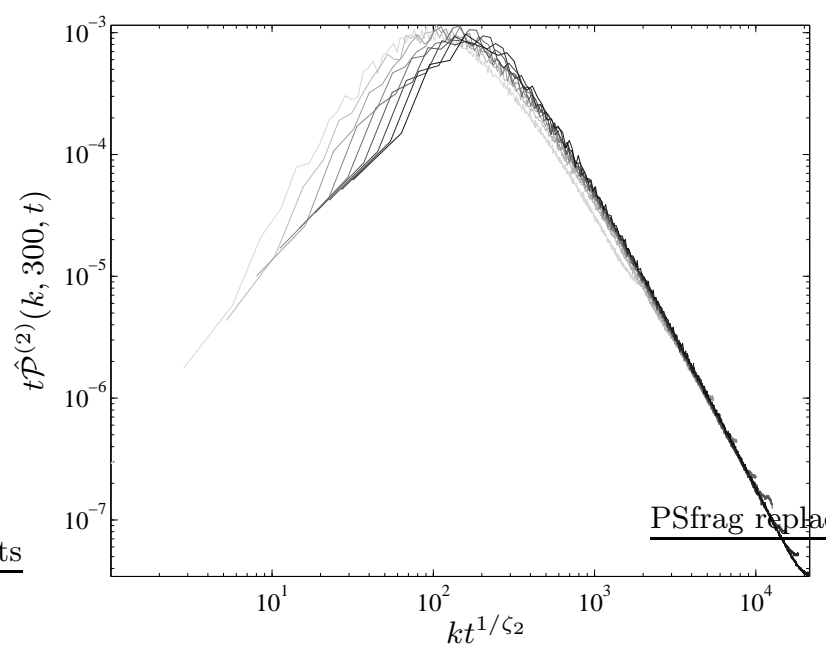

FIG. 5: $t \hat{\mathcal{P}}^{(2)}(k, 300, t)$ for 10 different times (light grey earliest dark grey last)

shown in Fig. 11 in different coordinates, multiplied by $t$ and as a function of

$$
\hat{k}=k t^{1 / \zeta_{2}} .
$$

The quality of the data collapse appears to strongly support the proposed scaling form. We note that the data collapse is superior on the right of the maximum, and less convincing at its left. We believe that this stems from two reasons. First, there is better statistics for the right part of the curve, simply because it belongs to larger $k$ vectors where the angular average is more extensive. Second, the left part of the curve is more sensitive to the finite size effects, particularly to the fact that the driving velocity field loses its scaling form close to $L$. With all the limitations of the numerical simulations we consider the data collapse as very satisfactory.

If the prediction for the 2-point function holds, then we will have for the time dependent integral:

$$
\int_{0}^{\infty} \hat{\mathcal{P}}^{(2)}\left(k, k^{\prime}, t\right) d k \approx \frac{1}{t^{1+1 / \zeta_{2}}} \int_{0}^{\infty} H^{(2)}(\hat{k}) d \hat{k} \propto \frac{1}{t^{1+1 / \zeta_{2}}}
$$

In Fig. 6] We show that indeed after an initial period the integral settles on a scaling form consistent with Eq. (61).

Using the form of the propagator we can establish the existence of a left zero mode of the 2-point propagator. Integrating over the two sides of Eq. (12) we have a time independent expression on the left hand side. We therefore expect that the weighed integral of the propagator with the function $Z^{(2)}$ will be constant:

$$
\begin{aligned}
& \int Z^{(2)}(\boldsymbol{k}) \mathcal{P}^{(2)}\left(\boldsymbol{k}, \boldsymbol{k}^{\prime}, t\right) d^{2} \boldsymbol{k} \\
& \propto \int_{0}^{\infty} k^{-2-\zeta_{2}} \frac{H^{(2)}\left(k t^{1 / \zeta_{2}}\right)}{t} k d k=\int_{0}^{\infty} \frac{H(\hat{k})}{\hat{k}^{1+\zeta_{2}}} d \hat{k}=\text { const }
\end{aligned}
$$

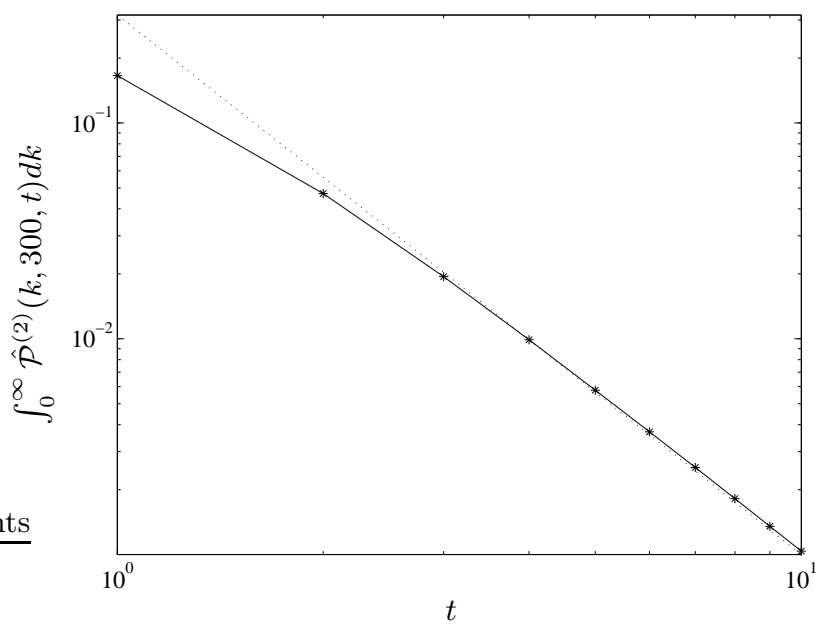

FIG. 6: The integral of the 2-point propagator, $\int_{0}^{\infty} \hat{\mathcal{P}}^{(2)}(k, 300, t) d^{2} k$, (solid line) as a function of time. The dotted line is the expected $t^{-\left(1+1 / \zeta_{2}\right)}$. (time is measured in this figure, and in the ones that follow in units of $\tau_{30}$ the eddy turnover time of $k=30$ )

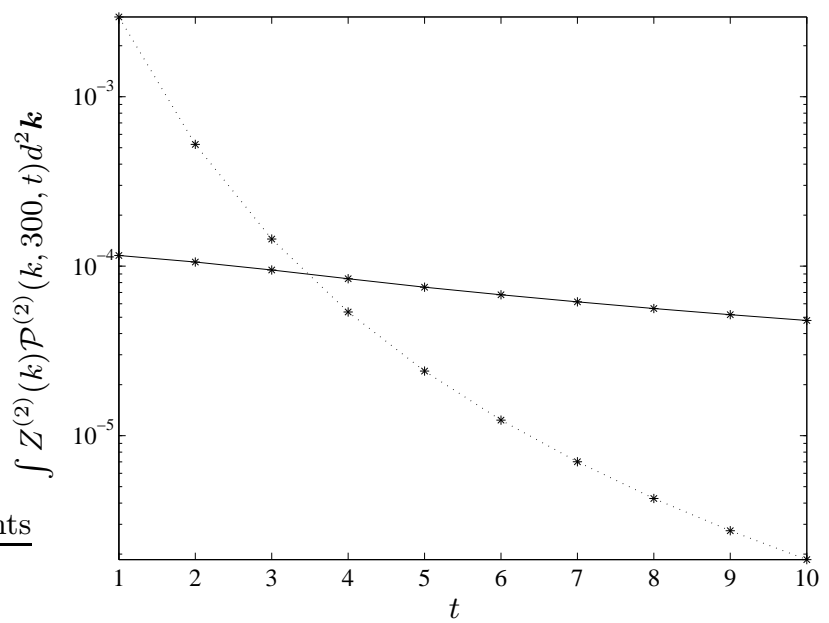

FIG. 7: The integral of the 2-point propagator $P^{(2)}(k, 300, t)$ weighed by the left zero mode $Z^{(2)}(k)$ (solid line), compared to the integral of the un-weighed second order structure function in dotted line.

where we have used the fact that in an isotropic 2dimensional systen we have $Z^{(2)}(\boldsymbol{k}) \sim F^{(2)}(\boldsymbol{k}) \propto k^{-2-\zeta_{2}}$. We note that the constancy of this integral should be judged on the background of the decaying function, as done in Fig. 7 We see that while the 2nd order structure function decays over three orders of magnitude, the "constant" objects changes by a factor of 2 . The lack of constancy can be attributed to the sensitivity to the outer scale as seen in the data collapse in Fig. (5). If the collapse on the left side of the curve were perfect, so would be the constancy of the weighted integral. Note that in the calculation we have employed $\zeta_{2}=0.67$ in agreement with [7] 

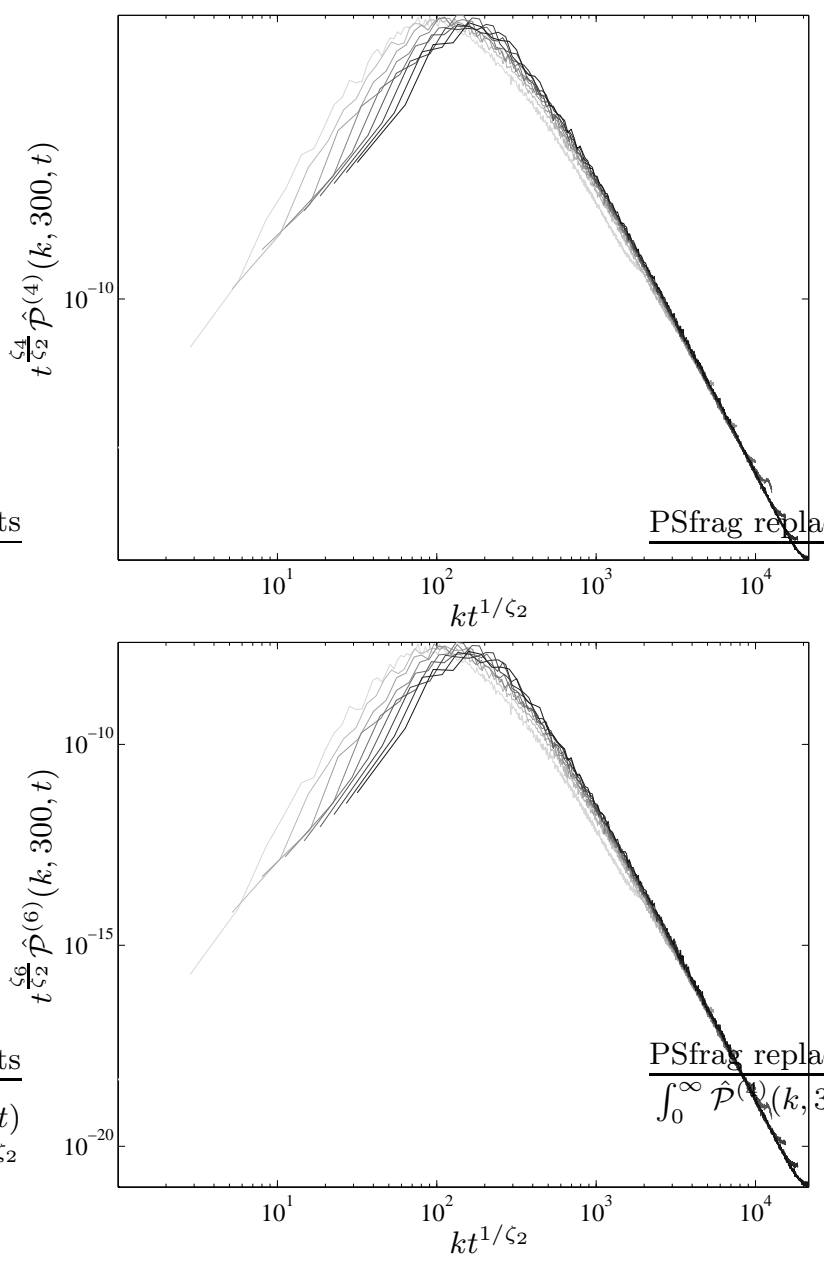

FIG. 8: $t^{\frac{\zeta_{4}}{\zeta_{2}}} \hat{\mathcal{P}}^{(4)}(k, 300, t)$ (upper panel) and $t^{\frac{\zeta_{6}}{\zeta_{2}}} \hat{\mathcal{P}}^{(6)}(k, 300, t)$ (lower panel) for 10 different times (light grey earliest dark grey last)

\section{B. analysis of the multi-point propagators}

Examining Eq.(58), we expect that for long times the functional form of the propagator for the fused correlation function defined in Eq.(22) is:

$$
\hat{\mathcal{P}}^{(2 N)}\left(k, k^{\prime}, t\right) \propto \frac{1}{t^{\zeta_{2 N} / \zeta_{2}}} H^{(2 N)}\left(k t^{1 / \zeta_{2}}\right),
$$

where $\zeta_{2 N}$ is the leading scaling exponent for the $2 N^{\prime}$ th correlation function. In Fig. 8 we demonstrate the data collapse obtained by assuming this form for the 4 'th and 6 'th order propagators. We notice the same excellent collapse on the right hand side of the function as in the 2nd order propagator. Also the problems with the outer scale show up in a similar manner, giving less than impressive collapse of the left hand part of the function. For the present data collapse we employed simple scaling $\zeta_{2 N}=N \zeta_{2}$; our data does not support strongly anomalous exponents.

Using the form of the fused multi-point correlators we
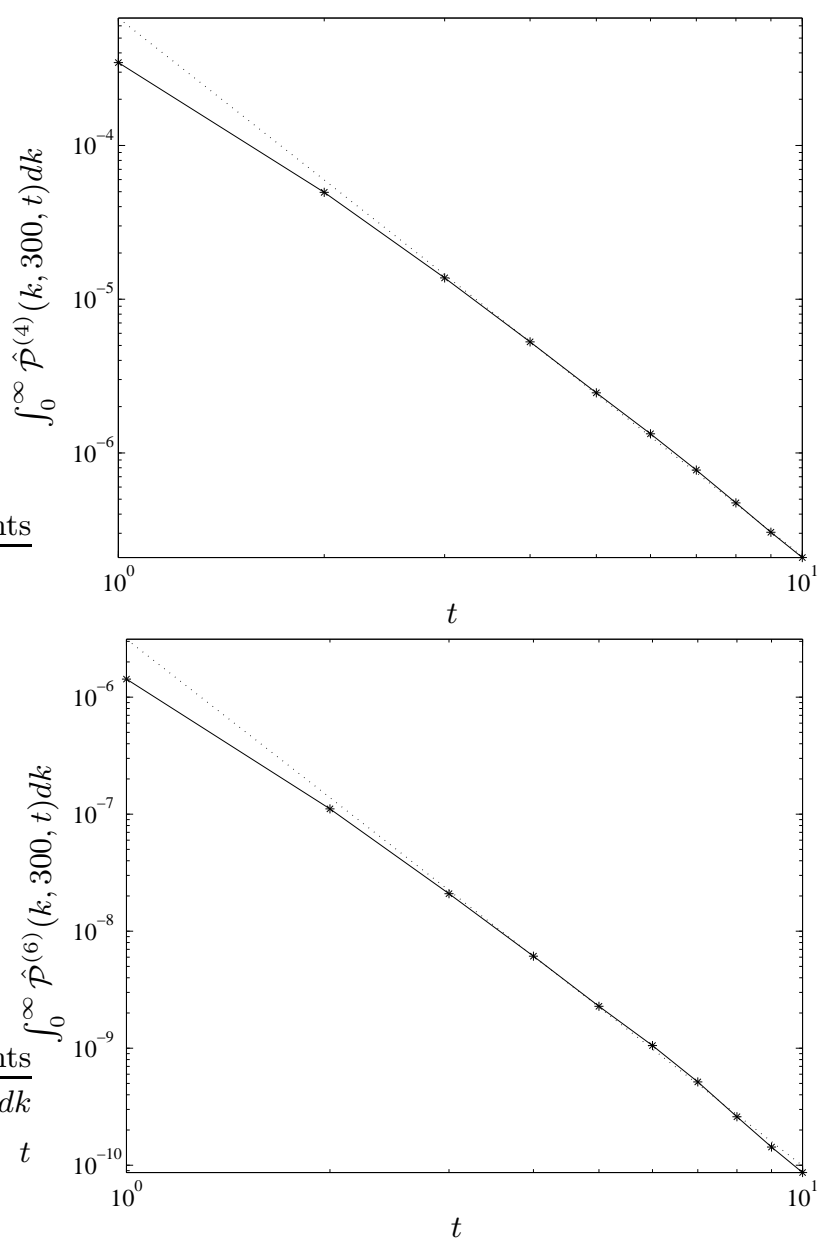

FIG. 9: The integral of the 4-point (upper panel) and 6-point (lower panel) propagators in solid line, $\int_{0}^{\infty} \hat{\mathcal{P}}^{(2 m)}(k, 300, t) d^{2} k$, compared with the expected $t^{-\left(1+\zeta_{2 N}\right) / \zeta_{2}}$, in the dotted line.

can again predict the time behavior of their integral:

$$
\begin{aligned}
\int_{0}^{\infty} \hat{\mathcal{P}}^{(2 N)}\left(k, k^{\prime}, t\right) d k & \approx \frac{1}{t^{\left(1+\zeta_{2 N}\right) / \zeta_{2}}} \int_{0}^{\infty} H(\hat{k}) d \hat{k} \\
& \propto \frac{1}{t^{\left(1+\zeta_{2 N}\right) / \zeta_{2}}}
\end{aligned}
$$

In Fig. 9 the time dependence of the integrals is plotted, showing agreement of similar quality to Fig. 6] As in the 2-point case, weighing the fused multi-point functions by the appropriate multi point fused steady state correlators should yield a constant:

$$
\begin{aligned}
& \int Z^{(2 N)}(k) \hat{\mathcal{P}}^{(2 N)}\left(k, k^{\prime}, t\right) d^{2} \boldsymbol{k} \\
& \propto \int_{0}^{\infty} \frac{H^{(2 N)}\left(k t^{1 / \zeta_{2}}\right)}{k^{2+\zeta_{2 N}} t^{\zeta_{2 N} / \zeta_{2}}} k d k=\int_{0}^{\infty} \frac{H^{(2 N)}(\hat{k})}{\hat{k}^{1+\zeta_{2 N}}} d \hat{k}=\mathrm{const}
\end{aligned}
$$

where $Z^{2 N}(k) \propto k^{-2-\zeta_{2 N}}$. The quality of the constancy is demonstrated in Fig. 10] Again finite size effects lead to some decrease in time of these weighted objects, which 

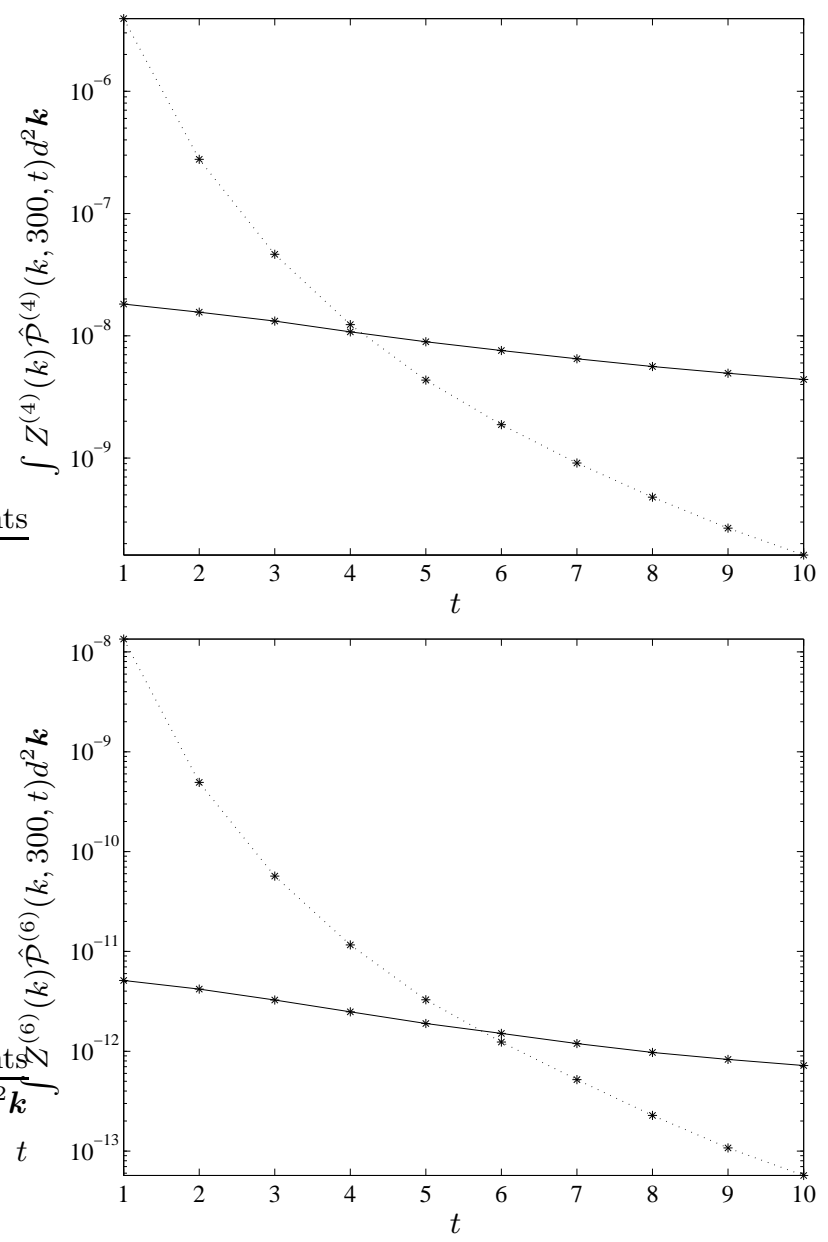

FIG. 10: The integral of the 4-point propagator $P^{(4)}(k, 300, t)$ (upper panel) and the 6-point propagator $P^{(6)}(k, 300, t)$ weighed by the left zero modes $Z^{(4)}(k)$ and $Z^{(6)}(k)$ respectively in solid line, compared to the integral of the un-weighed object in dotted line

nevertheless is very much reduced compared to the decaying correlation functions.

\section{CONCLUDING REMARKS}

We have demonstrated in this paper that the generic advection of a passive scalar by a velocity field that obeys the Navier-Stokes equations can be discussed in terms of Eulerian Statistically Preserved Structures. By initiating a decay with delta-function initial conditions (concentrated on $k=300$ ) we have found numerically the corresponding columns of the time-dependent propagators for the 2nd, 4th and 6th order correlation functions (where for the 4th and 6th order objects we considered partial ("fused") information). Note that in contrast to the Lagrangian formulation of Statistically Preserved Structures [3], for which there is no Preserved Structure corresponding to the 2nd order correlation, in the Eulerian formulation such an object exists and had been analyzed explicitly. We have used a simple (non-generic) model of passive scalar advection to guess the analytic scaling form of the propagators in the generic problem. The test for the relevance of this guessed form is the data collapse shown in Fig. 5 and 8. The guessed time dependence appears to be in close correspondence with the data as shown in Figs. 6 and 9.

The analytic forms of the propagators predict the existence of eigen-modes with eigenvalue 1 (Statistically Preserved Structures). We believe that this is the first demonstration of Eulerian Statistically Preserved Structures in a generic flow. The numerical evidence for the constancy of the latter is encouraging, if not fully conclusive, as seen in Figs. 7 and 10. We attributed the (relatively small) decrease in amplitude of the putative Statistically Preserved Structures to the less than perfect data collapse at the largest scales (smallest $k$-vectors) that are seen in Figs. 5 and 8. These in turn stem from the intervention of the outer scale in our scaling range, a boundary effect that we did not succeed to eliminate in our modest-size simulations. It would be interesting to see whether larger 2-d simulations could remove this finitesize effect to demonstrate conclusively the constancy of the Statistically Preserved Structures.

In concluding we wish to point out an additional benefit to the present formulation. Usually in modeling turbulent advection it is customary to resort to dubious concepts such as 'turbulent diffusion' in order to write a diffusion equation for the correlation functions. The present approach indicates a much better procedure, i.e. to find, for a given turbulent field, the form of the propagator which can be then used to provide analytic predictions for any initial $(t=0)$ correlation function. This procedure may be quite attractive for more complex hydrodynamic flows with unusual boundaries or coherent structures. We believe that when the turbulence is sufficiently well developed scaling forms for the propagator will exist, and once found can be used to solve efficiently any statistical initial value problem.

\section{Acknowledgments}

We thank Antonio Celani for providing us with his code for DNS of 2-dimensional turbulent advection. This work had been supported in part by the European Commission under a TMR grant, the Minerva Foundation, Munich, Germany, and the Naftali and Anna Backenroth-Bronicki Fund for Research in Chaos and Complexity. 
[1] A.S. Monin and A.M. Yaglom, Statistical Fluid Mechanics, vol. 1, chapter 5, (MIT, Cambridge 1979).

[2] D. Bernard, K. Gawedzki, A. Kupiainen, J.Stat. Phys 90, 519 (1998)

[3] A. Celani and M. Vergassola, Phys. Rev. Lett. 86, 424 (2001).

[4] I. Arad, L. Biferale, A. Celani, I. Procaccia and M. Vergassola, Phys. Rev. Lett. 87, 164502 (2001).
[5] Y. Cohen, T. Gilbert and I. Procaccia, Phys. Rev. E, 65, 026314 (2002).

[6] R. Benzi, L. Biferale and A. Wirth, Phys. Rev. Lett. 78, 4926 (1997)

[7] A. Celani, A. Lanotte, A. Mazzino and M. Vergassola, Phys. Rev. Lett. 84, 2385 (2000). 